\title{
A new selective blood agar medium for Streptococcus pyogenes and other haemolytic streptococci
}

\author{
E. J. L. LOWBURY, A. KIDSON, AND H. A. LILLY \\ From the Medical Research Council Industrial Injuries and Burns Research Unit, \\ Birmingham Accident Hospital
}

SYNOPSIS Horse blood agar containing polymyxin B sulphate, neomycin sulphate, and fusidic acid inhibited the growth of Staph. aureus, Ps. pyocyanea, Proteus mirabilis, E. coli, and Klebsiella pneumoniae but allowed good growth of, and haemolysis by, Str. pyogenes.

In a comparison with blood agar, blood $4 \%$ agar, and gentian violet blood agar, the selective medium (P.N.F.) yielded a significantly higher proportion of streptococci than the other media, both by aerobic and by anaerobic culture, from burn swab extracts deliberately contaminated with Str. pyogenes; P.N.F. culture was more effective with dilute than with heavy inocula of Str. pyogenes, allowing from three to seven times as many recoveries of Str. pyogenes from swabs contaminated with $10^{-3}$ dilution of streptococcal cultures than cultures of the same material on the other media.

Haemolytic streptococci of groups A, C, D, G, and others were isolated by aerobic culture from burns in a consecutive series of 1,277 swabs more often on P.N.F. medium than on blood $4 \%$ agar.

Viable counts of 12 strains (including 11 different serological types) of Str. pyogenes showed some reduction in the numbers of colonies compared with counts on blood agar, and some strains grew more slowly on P.N.F. medium. These limitations, however, were offset and outweighed by the higher final yield of streptococci on the selective medium.

Streptococcus pyogenes is commonly isolated in mixed culture from burns and from the upper respiratory tract. When the growth of other bacteria is abundant, it may be difficult or impossible to detect or pick colonies of streptococci on unselective blood agar, especially when the streptococci are present in small numbers. Anaerobic culture (Fry, 1933; Williams and Miles, 1945) and the use of gentian violet in blood agar (Garrod, 1933, 1942; Francis, 1941) have been found useful in selecting streptococci from mixed cultures. The use of concentrated agar, which was introduced by Hayward and Miles (1943) to inhibit the swarming of Proteus and certain clostridia, is another valuable aid in the isolation of streptococci, especially from wounds; the bacterial flora of burns have for many years been examined in this Unit by culture on blood agar plates containing 4 to $6 \%$ agar (Lowbury, 1960). Each of these methods, however, has limited selective power, and it may be difficult or impossible to pick colonies, even when they are seen, from culture plates where Gram-negative bacilli predominate.

Received for publication 5 December 1963.
Str. pyogenes is relatively resistant to polymyxin, neomycin, and fusidic acid, three antibiotics which are highly active against different but overlapping ranges of the other bacteria commonly found in burns and in the upper respiratory tract. In this paper we give a preliminary report on a selective medium containing these three antibiotics, and compare the isolation on it and on some other media of haemolytic streptococci in the presence of the mixed flora of burns.

\section{COMPOSITION OF THE SELECTIVE MEDIUM}

GROWTH OF STR. PYOGENES The highest concentrations of neomycin sulphate, polymyxin B sulphate, and fusidic acid in $5 \%$ horse blood agar (P.N.F. medium) which allowed a good growth and haemolysis by two strains of Str. pyogenes (NT 276 from a throat swab and 62/8459 from a burn) were determined. Viable counts were obtained by the method of Miles and Misra (1938) on the medium containing the antibiotics and without them. Tests were made first with a range of dilutions of fusidic acid, then with the highest non-inhibitory concentrations of 
TABLE I

ASSESSMENT OF OPTIMUM CONCENTRATION OF ANTIBIOTICS IN BLOOD AGAR FOR GROWTH AND HAEMOLYSIS OF A STRAIN OF STREPTOCOCCUS PYOGENES (NT 276)

Antibiotics in Blood Agar

Highest Concentration ( $\mu$ g. per $\mathrm{ml}$.) Allowing Lowest Concentration ( $\mu \mathrm{g}$. per ml.) Allowing

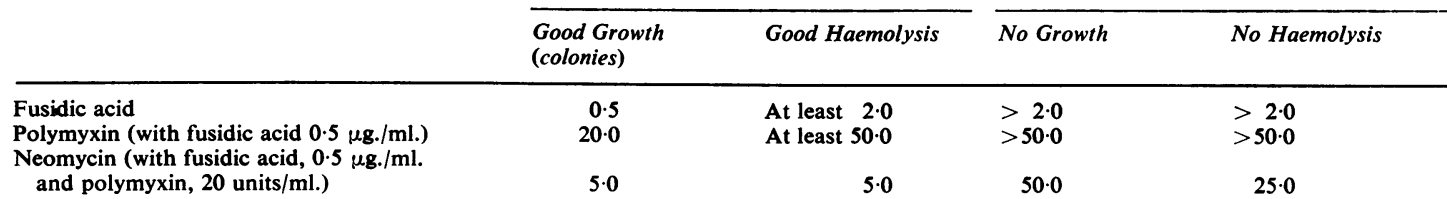

fusidic acid and a range of dilutions of polymyxin, and finally with the chosen concentrations of fusidic acid and polymyxin and a range of dilutions of neomycin. Plates were examined after aerobic incubation for 24 hours.

Table I shows the results of this series of tests with the strain NT 276. A medium containing the maximum concentration of the antibiotics allowing growth and haemolysis of strain NT 276 was then tested for viable counts of 11 strains of the Str. pyogenes of different serological types. Some of these strains were slightly more sensitive to the antibiotics than strain NT 276; the highest concentrations of the antibiotics in blood agar which allowed growth and recognition of the more sensitive streptococci were (1) polymyxin B sulphate, 17 units per $\mathrm{ml}$., (2) neomycin sulphate, $4 \cdot 25 \mu \mathrm{g}$. per $\mathrm{ml}$., and (3) fusidic acid, $0.5 \mu \mathrm{g}$. per $\mathrm{ml}$. On blood agar containing these concentrations of the antibiotics (P.N.F. medium), all the strains of Str. pyogenes grew satisfactorily and showed good haemolysis, though some strains showed very small colonies after 18 hours' incubation. The viable counts of Str. pyogenes were somewhat smaller on the selective than on unselective blood agar (Table II); this disadvantage, however, was outweighed by the strong selective action of the medium.

\section{TABLE II}

VIABLE COUNTS OF 12 STRAINS OF STR. PYOGENES INOCULATED ON P.N.F. MEDIUM AND ON BLOOD AGAR

\begin{tabular}{|c|c|c|c|}
\hline \multicolumn{2}{|c|}{ Streptococcus } & \multicolumn{2}{|c|}{$\begin{array}{l}\text { Viable Counts from } 0.1 \mathrm{ml} \text {. } \\
\text { of Inoculum }\end{array}$} \\
\hline Strain & Type & $\begin{array}{l}\text { On P.N.F. } \\
\text { Medium }\end{array}$ & On Blood Agar \\
\hline 8198 & 1 & 800 & 1,600 \\
\hline 100065 & 5 & 14 & 18 \\
\hline 8326 & 4 & 6,000 & 12,000 \\
\hline 8191 & 3 & 8,000 & 17,000 \\
\hline 100068 & 11 & 120,000 & 300,000 \\
\hline 8302 & 6 & 120,000 & 240,000 \\
\hline 8320 & 18 & 50,000 & 240,000 \\
\hline 100085 & 12 & 200,000 & $1,400,000$ \\
\hline 8322 & 2 & 2,000 & 6,000 \\
\hline 8194 & 19 & 9,000 & 40,000 \\
\hline 8305 & 24 & 20,000 & 36,000 \\
\hline NT 276 & 12 & 2,600 & 5,400 \\
\hline
\end{tabular}

INHIBITION OF BACTERIA OTHER THAN STREPTOCOCCI The P.N.F. medium was tested for its ability to inhibito the growth of bacteria likely to be present together with Str. pyogenes in infective lesions. Undiluted $\omega$ broth cultures of strains of Staph. aureus $(63 / 6321$, N 63/1621), Pseudomonas pyocyanea (63/6275), Escherichia coli (63/6347), Proteus mirabilis (63/6423), and오 Klebsiella pneumoniae $(63 / 6348)$ were inoculated onP.N.F. medium; after incubation under aerobic and anaerobic conditions there was no growth of Staph. aureus, and the growth of Gram-negative $\overrightarrow{0}$ bacilli was strongly inhibited; lower concentrations + of neomycin allowed Proteus mirabilis to swarm ando Ps. pyocyanea to grow more freely.

Mixtures of these cultures with a range of dilutions of a culture of Str. pyogenes (NT 276) were inoculated. in duplicate on plates of P.N.F. medium, horsed blood agar (1.5\% agar), blood agar containing gentian violet (1 in 500,000), and horse blood agaro containing $4 \%$ agar; one culture plate of each medium was incubated aerobically and one anaerobically. There was good selective growth of Str pyogenes on P.N.F. medium from mixed inoculao with Staph. aureus and with Gram-negative bacillio (Figs. 1, 2, and 3). Of the other methods tested, anaerobic culture improved the isolation of Str. pyogenes by inhibiting growth of the other bacteria; $4 \%$ agar also made the isolation of streptococcio easier by preventing the swarming of Proteus and reducing the size of colonies. There was diffuse haemolysis by Gram-negative bacilli on the un selective media incubated aerobically. The gentian ${ }^{\circ}$ violet medium, which is strongly inhibitory towards 0 staphylococci, showed little selective value in the presence of Gram-negative bacilli (in particularo Proteus, which swarmed vigorously on aerobico culture and also to some extent on anaerobic culture on this medium).

PREPARATION OF THE MEDIUM Hartley's digest agaro (Oxoid) was melted and cooled to about $50^{\circ} \mathrm{C}$. Solutions of the antibiotics were added separately and mixed intoD the medium together with horse blood. Plates of this medium were kept in the refrigerator and tested for growth of Str. pyogenes and inhibition of other bacteriag 



FIGS. 1, 2, and 3. Plates of three media (blood agar, gentian violet blood agar, and P.N.F.) inoculated with (a) on left, Streptococcus pyogenes and Staphylococcus aureus and $(b)$ on right, Streptococcus pyogenes with a mixture of Proteus mirabilis, Pseudomonas pyocyanea, Escherichia coli, and Klebsiella pneumoniae. The plates were incubated aerobically at $37^{\circ} \mathrm{C}$. for 24 hours. Gentian violet blood agar was selective for Str. pyogenes in presence of Staph. aureus only; P.N.F. medium was selective for the streptococci in mixtures with staphylococci and with Gram-negative bacilli.

for periods up to four weeks; there was no loss of selectiveness in that time.

Medium prepared by adding concentrated mixtures of the three antibiotics allowed growth with swarming of Proteus and appeared to be deficient in selective power; for this reason concentrated solutions of the three antibiotics for use in this medium were stored separately. Since agar plates retain their activity on storage at $4^{\circ} \mathrm{C}$. for at least four weeks, it should be possible to keep under these conditions bottles of nutrient agar containing polymyxin, neomycin, and fusidic acid as a base for the selective medium.

TRIALS OF THE SELECTIVE MEDIUM IN THE ISOLATION OF STREPTOCOCCI FROM BURNS

RECOVERY ON DIFFERENT MEDIA OF STR. PYOGENES ADDED TO SWAB EXTRACTS Swabs from 200 burns were extracted by rubbing thoroughly in a small volume $(0.2 \mathrm{ml}$.) of digest broth. One drop $(0.02 \mathrm{ml}$.) of an undiluted overnight broth culture of Str. pyogenes (NT 276) was added to each of 50 tubes containing swab extracts; similar additions to sets of 50 tubes of swab extract were made with $10^{-1}, 10^{-2}$, and $10^{-3}$ dilutions of the culture of Str. pyogenes. The extract was inoculated on two plates each of the following media: (1) P.N.F. medium; (2) blood agar (4\% New Zealand agar); (3) blood agar (1.5\% agar); (4) blood agar (1.5\% agar) containing 1 in 500,000 gentian violet.

One plate of each pair was incubated aerobically, the other anaerobically. Plates were examined with the naked eye and with a hand lens after 18 hours' incubation. Small haemolytic colonies were picked, examined for morphology and tested by Lancefield's method with streptococcal antiserum of group A.

Results Table III shows that with each dilution of Str. pyogenes the largest proportion of cultures from which a streptococcus of group A was recovered, either aerobically or anaerobically, was among those plated on P.N.F. medium; anaerobic conditions and growth on $4 \%$ agar also increased the frequency of isolation of the streptococcus, especially when the inoculum of streptococci was large, and the yield of Str. pyogenes in the series

\section{TABLE III}

RECOVERY OF ADDED STREPTOCOCCUS PYOGENES (NT 276) FROM SUSPENSIONS OF BURNS SWABS ON DIFFERENT MEDIA AFTER 18 HOURS' AEROBIC AND ANAEROBIC CULTURE

Dilutions of Added Str. pyogenes

Number of Swabs from which Str. pyogenes was Isolated on

Swabs Examined

\begin{tabular}{|c|c|c|c|c|c|c|c|c|c|}
\hline \multirow{3}{*}{ Str. pyogenes } & \multirow{2}{*}{\multicolumn{2}{|c|}{ Blood Agar }} & \multirow{2}{*}{\multicolumn{2}{|c|}{ Blood 4\% Agar }} & \multirow{2}{*}{\multicolumn{2}{|c|}{$\begin{array}{l}\text { Gentian Violet Blood } \\
\text { Agar }\end{array}$}} & \multirow{2}{*}{\multicolumn{2}{|c|}{ P.N.F. Medium }} & \\
\hline & & & & & & & & & \\
\hline & Aerobic & Anaerobic & Aerobic & Anaerobic & Aerobic & Anaerobic & Aerobic & Anaerobic & \\
\hline $\begin{array}{l}\text { Undiluted } \\
10^{-1} \\
10^{-2} \\
10^{-3}\end{array}$ & $\begin{array}{r}16 \\
7 \\
5 \\
2\end{array}$ & $\begin{array}{r}38 \\
7 \\
7 \\
4\end{array}$ & $\begin{array}{r}31 \\
20 \\
7 \\
5\end{array}$ & $\begin{array}{r}45 \\
26 \\
12 \\
7\end{array}$ & $\begin{array}{r}18 \\
10 \\
7 \\
3\end{array}$ & $\begin{array}{r}36 \\
10 \\
8 \\
3\end{array}$ & $\begin{array}{l}38 \\
35 \\
29 \\
15\end{array}$ & $\begin{array}{l}47 \\
37 \\
34 \\
17\end{array}$ & $\begin{array}{l}50 \\
50 \\
50 \\
50\end{array}$ \\
\hline
\end{tabular}


with undiluted streptococci from anaerobic $4 \%$ agar cultures was slightly greater than that from aerobic culture on P.N.F. medium. With streptococci added at dilutions $10^{-1}, 10^{-2}$, and $10^{-3}$, however, P.N.F. medium gave a higher yield of streptococci either aerobically or anaerobically than aerobic or anaerobic culture on any of the other media. With streptococci added at $10^{-1}$ dilution, P.N.F. medium gave significantly higher yields of streptococci than gentian violet blood agar (aerobic: $\chi^{2}=23.2, P<0.001 ;$ anaerobic; $\chi^{2}=27.2$, $\mathrm{P}<0.001$ ), blood agar with $4 \%$ agar (aerobic: $\chi^{2}=8.0, \mathrm{P}<0.01$; anaerobic: $\chi^{2}=4.3, \mathrm{P}<0.05$ ) and blood agar with $1.5 \%$ agar (aerobic: $\chi^{2}=30.0$, $\mathrm{P}<0.001$; anaerobic: $\left.\chi^{2}=34.2, \mathrm{P}<0.001\right)$. With higher dilutions of added streptococci the differences between P.N.F. medium and the other media were still greater, and the advantages of anaerobic culture less obvious.

ISOLATION OF HAEMOLYTIC STREPTOCOCCI FROM A SERIES OF SWABS FROM BURNS A series of 1,277 consecutive swabs from burns of patients in the Burns Unit was inoculated both on blood agar with $4 \%$ agar (the medium used for routine culture of burns) and on P.N.F. medium; the order of inoculating the two media was reversed with successive swabs, so that an equal number of swabs should be plated first and second on each medium.

Both media were incubated aerobically at $37^{\circ} \mathrm{C}$. and examined after 18 hours' and again after 42 hours' incubation; cultures on P.N.F. medium which showed no haemolytic streptococcal colonies after 42 hours' incubation were returned to the incubator for a third night and examined after 60 hours' incubation.

Small haemolytic colonies were examined for morphology and grouped by Lancefield's method with antisera of groups A, C, D, and G.

Results Table IV shows the isolation of different groups of streptococci from 1,277 burn swabs plated on the two media. Each group and also the unclassified streptococci were isolated more frequently from P.N.F. medium than from blood agar; the differences reached the level of statistical significance in the case of Str. pyogenes (group A) and of group $\mathrm{D} \frac{\text { (ै) }}{\vec{\sigma}}$ streptococci, and the difference in the total positives (117 on blood agar and 184 on P.N.F. medium) was $\overrightarrow{\vec{F}}$ highly significant $\left(\chi^{2}=16 \cdot 4, \mathrm{P}<0 \cdot 001\right)$. There was, however, some delay in the detection of certain $\frac{C}{0}$ streptococci of groups A and D; indeed, the former $\bar{c}$ were isolated after 18 hours more frequently on $\widehat{\Phi}$

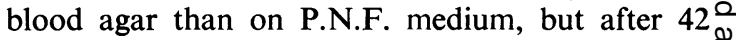
hours isolations of Str. pyogenes on P.N.F. and on ${ }^{\infty}$ blood agar were in the ratio of 3 to 2 and after $\vec{O}$ 60 hours nearly twice as many isolations were obtained from the selective medium. Unselective ${ }_{\sigma}^{\omega}$ blood agar yielded no more positives when examined after 42 hours' than after 18 hours' incubation, and? no further isolations could be expected on still more $\vec{v}$ prolonged incubation, as the medium often became $\omega$ overgrown with Gram-negative bacilli and diffusely haemolysed.

\section{DISCUSSION}

A selective medium for haemolytic streptococci $\overrightarrow{0}$ (including Str. pyogenes) is made possible through the relatively weak inhibition of these organisms by three antibiotics, namely, polymyxin, neomycin, and fusidic acid. Our preliminary studies have shown a higher frequency of isolation from burns of haemolytic streptococci of groups A, C, D, and G and of some other streptococci on such a medium (P.N.F.) than on a standard blood agar medium, and its $\overrightarrow{\vec{O}}$ superiority to gentian violet blood agar in the 3 isolation of a strain of group A streptococcus from the mixed flora of burns has also been demonstrated.

The concentration of the antibiotics in this blood containing medium which will allow good growth: and haemolysis of Str. pyogenes is, in general, sufficient to inhibit completely the growth of staphylococci but rather too low to produce more than a partial inhibition of some of the other organisms that are apt to conceal the streptococcus in cultures from sites of mixed infection. Another limitation is the rather slow growth of some strains

TABLE IV

ISOLATION OF HAEMOLYTIC STREPTOCOCCI FROM 1,277 BURNS SWABS ON P.N.F. MEDIUM AND ON BLOOD $4 \%$ AGAR BY AEROBIC CULTURE

\begin{tabular}{|c|c|c|c|c|c|c|c|c|}
\hline \multirow{3}{*}{$\begin{array}{l}\text { Streptococcus of } \\
\text { Group }\end{array}$} & \multicolumn{5}{|c|}{ Swabs from which Haemolytic Streptococci were Isolated on } & \multirow{3}{*}{$\begin{array}{l}\text { Total No. of } \\
\text { Swabs Positive } \\
\text { (either medium) }\end{array}$} & \multirow{2}{*}{\multicolumn{2}{|c|}{$\begin{array}{l}\text { Significance Tests } \\
(\text { for } \% \text { swabs }+ \text { ) }\end{array}$}} \\
\hline & \multicolumn{2}{|c|}{ Blood Agar 4\% } & \multicolumn{3}{|c|}{ P.N.F. Medium } & & & \\
\hline & By 18 Hours & By 42 Hours & By 18 Hours & By 42 Hours & By 60 Hours & & & \\
\hline $\mathbf{A}$ & 20 & 20 & 13 & 30 & 37 & 37 & $6 \cdot 0$ & $<0.02$ \\
\hline C & 21 & 21 & 32 & 32 & 32 & 34 & $2 \cdot 3$ & $>0.05$ \\
\hline D & 52 & 52 & 65 & 76 & 76 & 85 & $4 \cdot 3$ & $<0.05$ \\
\hline G & 19 & 19 & 25 & 25 & 25 & 26 & 0.6 & $>0.05$ \\
\hline Others & 5 & 5 & 14 & 14 & 14 & 15 & $3 \cdot 6$ & $>0.05$ \\
\hline Total & 117 & 117 & 149 & 177 & 184 & 197 & $16 \cdot 4$ & $<0.001$ \\
\hline
\end{tabular}


of Str. pyogenes growing from swabs on the selective medium, making it necessary to examine culture plates after 18 and 42 hours' and even 60 hours' incubation to obtain the full advantage of the method. These disadvantages, however, are offset by the consistently and significantly higher proportion of positive swabs obtained on the selective medium. By its use we have, for example, found that some wounds thought from conventional tests to have been cleared of Str. pyogenes by antibiotic treatment were still infected. Streptococcal colonies have often been easy to pick from P.N.F. medium when other media inoculated with the same material were too heavily colonized by coliform bacilli and other organisms for the haemolytic colonies to be picked, even when they are seen. To obtain optimal conditions for the isolation of Str. pyogenes from scanty or heavy inocula in pure or mixed culture it would seem desirable to inoculate both P.N.F. medium and a medium which has no inhibitory effect on the streptococci.

These studies have confirmed the selective value of anaerobic culture and of culture on a medium containing $4 \%$ agar; though less than that of P.N.F. medium, this selective action is useful, particularly when the streptococci are numerous but mixed with even more numerous Gram-negative bacilli. The value of gentian violet in selecting Str. pyogenes from mixed cultures of streptococci and staphylococci was also shown. The selective effect of $4 \%$ agar probably depends not only on its inhibition of the swarming of Proteus but also on the apparent suppression of hydrogen peroxide production by the streptococci; this mechanism was considered as a possible explanation of the aerobic growth on $4 \%$ but not on $1.5 \%$ agar medium of an atypical tetracycline-resistant Str. pyogenes isolated from burns (Lowbury and Hurst, 1956).

The scope of the new medium cannot be adequately assessed yet. It is clearly more effective than the other media tested in the examination of burns for streptococci, and especially for the detection of small numbers of organisms (e.g., in tests on the effects of chemotherapy); the advantages in using P.N.F. medium are particularly striking on aerobic culture. Further studies are needed to determine whether this medium is more useful than others in the examination of flora of the upper respiratory tract, where Gram-negative bacilli are less common than they are in burns or other wounds.

We wish to thank Mr. R. Gill for the photographs.

\section{REFERENCES}

Francis, A. E. (1941). Lancet, 2, 159.

Fry, R. M. (1933). J. Path. Bact., 37, 337.

Garrod, L. P. (1933). St. Bart. Hosp. Rep., 66, 203. - (1942). Brit. med. J., 1, 290.

Hayward, N. J., and Miles, A. A. (1943). Lancet, 2, 116.

Lowbury, E. J. L. (1960). Brit. med. J., 1, 994. - and Hurst, L. (1956). J. clin. Path., 9, 59.

Miles, A. A., and Misra, S. S. (1938). J. Hyg. (Lond.), 38, 732. Williams, R. E. O., and Miles, A. A. (1945). J. Path. Bact., 57, 27. 